\title{
Different Regions Identification in Composite Strain-Encoded (C-SENC) Images Using Machine Learning Techniques
}

\author{
Abdallah G. Motaal $^{1}$, Neamat El-Gayar ${ }^{1,2}$, and Nael F. Osman ${ }^{1,3}$ \\ ${ }^{1}$ Center for Informatics Sciences, Nile University, Egypt \\ ${ }^{2}$ Faculty of Computers and Information, Cairo University, 12613 Giza, Egypt \\ ${ }^{3}$ Radiology Department, School of Medicine, Johns Hopkins University, USA \\ abdallah.motaal@nileu.edu.eg \\ \{nelgayar, nosman\}@nileuniversity.edu.eg
}

\begin{abstract}
Different heart tissue identification is important for therapeutic decision-making in patients with myocardial infarction (MI), this provides physicians with a better clinical decision-making tool. Composite Strain Encoding (C-SENC) is an MRI acquisition technique that is used to acquire cardiac tissue viability and contractility images. It combines the use of blackblood delayed-enhancement (DE) imaging to identify the infracted (dead) tissue inside the heart muscle and the ability to image myocardial deformation from the strain-encoding (SENC) imaging technique. In this work, various machine learning techniques are applied to identify the different heart tissues and the background regions in the C-SENC images. The proposed methods are tested using numerical simulations of the heart C-SENC images and real images of patients. The results show that the applied techniques are able to identify the different components of the image with a high accuracy.
\end{abstract}

\section{Introduction}

Imaging of the heart anatomy and function using magnetic resonance imaging (MRI) is an important diagnostic tool for heart diseases. Delayed-enhancement (DE) MRI after contrast agent administration is used to differentiate viable from infarcted myocardial tissue. Also, myocardial contractility pattern can be characterized from functional MR images. By combining the information from the viability and functional images, three different tissue types can be distinguished: 1) healthy myocardium; 2) infarcted myocardium; and 3) non-contracting, but viable, tissue, which could represent hibernating tissue [1]. The identification of the hibernating myocardium is very important as it is the tissue that will mostly benefit from revascularization [2]. Inversion-recovery imaging is considered the gold standard technique for acquiring DE MR Images [3]. The obtained image has T1-weighted contrast, and after the administration of the contrast agent, high signal intensity from infarcted myocardium is obtained. Strain-encoding (SENC) MR is used for directly imaging myocardial strain [4] where it is based on applying parallel planes of saturated magnetization to the cardiac tissue with initial tagging frequency, $\omega_{\mathrm{o}}$, which 
depends on the slice thickness and slice profile in the $z$-direction. Two images are then acquired with different demodulation frequencies, in the $z$-direction, from which a functional image of the heart is obtained [4]. Composite SENC have been developed, where it shows both functional and viability information of the heart in single acquisition [1].

Previously, a method is proposed to identify different heart tissues from MRI CSENC images using an unsupervised multi-stage fuzzy clustering technique. The method was based on sequential application of the fuzzy c-means (FCM) and iterative self-organizing data (ISODATA) clustering algorithms [5]. In a more recent work [6], a bayesian classifier is proposed to identify the background region (air), then the filtered tissue regions are classified into the different tissue types using fuzzy Cmeans clustering algorithm.

In this work, several classification and clustering techniques are proposed to identify the background and the different tissue types. Numerical simulations and real MR images of patients are used to validate the segmentation techniques, which show excellent results. The paper is organized as follows; section 2 briefly describes the CSENC. Section 3 describes the proposed system. Section 4 describes the used data sets, explains the details of the experiments conducted, and the results are presented. Finally, the paper is discussed and concluded in sections 5.

\section{Theory of C-SENC}

The SENC technique is used to measure the local strain of deforming tissues. In SENC MRI, the magnetization of the object under test at point $y$ and time $t$ is modulated in the slice-selection direction with a sinusoidal pattern of a spatial frequency, $\omega_{0}(y, t)$-which is initially uniform everywhere. Because of the contraction of the LV, myocardial deformation occurs, and the tag pattern moves and undergoes deformation that makes the tissue's new frequency, $\omega(\mathrm{y}, \mathrm{t})$, proportionally changing with the degree of deformation at the pixel $y$. The resulting image intensity at this pixel can be given by [4]:

$$
\mathrm{I}\left(\mathrm{y}, \mathrm{t} ; \omega_{\mathrm{T}}\right)=\int_{-\infty}^{\infty} \mathrm{M}(\mathrm{y}, \mathrm{z}, \mathrm{t}) \mathrm{S}(\mathrm{z}) \mathrm{e}^{-\mathrm{j} \omega_{\mathrm{T}} \mathrm{z}} \mathrm{dz},
$$

where $y$ is the pixel location, $\omega_{\mathrm{T}}$ is the tuning value, and $\mathrm{I}\left(\mathrm{y}, \mathrm{t} ; \omega_{\mathrm{T}}\right)$ is the signal intensity. The equation shows that the signal intensity at a certain point and time is the integral in the slice-selection direction of the longitudinal magnetization multiplied by the encode phase factor over the slice profile $S(z)$. Using some simplifications [4], the resulting image is approximated to

$$
I(y, t)=\rho(y, t) S\left(\omega_{T}-\omega(y, t),\right.
$$

where $\rho(y, t)$ represents the proton density of the voxel, and $S(\omega)$ is the Fourier transform of the slice profile. $\omega_{\mathrm{T}}$ is called the tuning frequency, which is determined during the image acquisition by an applied tuning gradient. It is noticed that the function $\mathrm{S}($.$) is shifted in proportion to the change in the tagging frequency, \omega$, which depends on the tissue deformation. Two images are acquired at two tuning 
frequencies, $\omega_{\mathrm{a}}$ and $\omega_{\mathrm{b}}$, and from these two images we can estimate the local frequency of the slice using the following relation [4]:

$$
\omega(\mathrm{y}, \mathrm{t})=\frac{\omega_{\mathrm{a}}\left|\mathrm{I}\left(\mathrm{y}, \mathrm{t} ; \omega_{\mathrm{a}}\right)\right|+\omega_{\mathrm{b}}\left|\mathrm{I}\left(\mathrm{y}, \mathrm{t} ; \omega_{\mathrm{b}}\right)\right|}{\mathrm{I}\left(\mathrm{y}, \mathrm{t} ; \omega_{\mathrm{a}}\right)+\mathrm{I}\left(\mathrm{y}, \mathrm{t} ; \omega_{\mathrm{b}}\right)},
$$

where $\mathrm{I}\left(\mathrm{y}, \mathrm{t} ; \omega_{\mathrm{a}}\right)$ and $\mathrm{I}\left(\mathrm{y}, \mathrm{t} ; \omega_{\mathrm{b}}\right)$ are the images acquired at tuning values $\omega_{\mathrm{a}}$ and $\omega_{\mathrm{b}}$ respectively. Since the strain is the change in length per unit length,

$$
\varepsilon(\mathrm{y}, \mathrm{t})=\frac{\Delta \mathrm{L}}{\mathrm{L}}=\frac{\mathrm{L}_{t}-\mathrm{L}_{1}}{\mathrm{~L}_{\mathrm{i}}}=\frac{\frac{1}{\omega_{t}}-\frac{1}{\omega_{\mathrm{o}}}}{\frac{1}{\omega_{\mathrm{o}}}},
$$

thus, the tissue strain at location $y$ and time $t$ can be estimated from [4]:

$$
\varepsilon(\mathrm{y}, \mathrm{t})=\left(\frac{\omega_{\mathrm{o}}}{\omega_{\mathrm{t}}}-1\right) \times 100,
$$

where $\omega_{\mathrm{o}}$ is the initial tagging frequency and $\omega_{\mathrm{t}}$ is the tags frequency at time $t$.

In C-SENC [1], the SENC pulse sequence is modified to acquire an image at the beginning of the acquisition with no-tuning to capture the signal from the recovering DC magnetization. Thus the first image differentiates between normal and infarcted myocardium because of their different longitudinal recovery rates after contrast agent injection, the second image, low-tune image, shows the static tissue, and the third image, high-tune image, shows the contracting tissue.

\section{Proposed System}

Several classifiers are used to classify the different image regions. First, an input vector, $x=\left[f_{1}, f_{2}, f_{3}, l\right]$, is constructed, where $f_{1}$ is a feature that represents the pixel intensity in the high-tune image, $f_{2}$ is a feature that represents the pixel intensity in the low-tune image, $f_{3}$ is a feature that represents the pixel intensity in the no-tune image, and $L$ is the label of the current pixel, where $L \in\{$ 'healthy', 'infarcted, 'hibernating', 'background'\} regions. Fig. 1 shows the input data.

\begin{tabular}{|c|c|c|c|}
\hline$f_{1}$ & $f_{2}$ & $f_{3}$ & $\iota$ \\
\hline H.T intensity & L.T intensity & N.T intensity & Label \\
\hline
\end{tabular}

Fig. 1. The input data vector

In case of using unsupervised learning clustering algorithm, simple K-means, a labeling stage is added after the clustering stage and the input data becomes 3-D only, as the fourth cell will be omitted. The labeling stage uses information from the average-pixel intensity in each identified cluster. The rules used in the labeling stage are usually verified by medical domain experts. Fig. 2 shows the proposed system. 


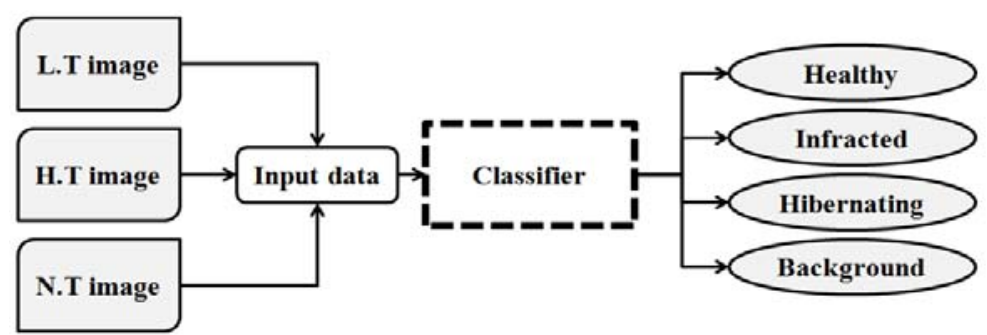

Fig. 2. The block diagram of the proposed system

\section{Experimental Results}

Simulated images with signal-to-noise ratio $21 \mathrm{~dB}$ are generated for C-SENC images. This data represents test data containing regions of healthy, infracted, hibernating tissues and background. The image is divided into four regions, where the upper quarter represents a healthy contracting tissue, the second quarter from above represents hibernating tissue, the third quarter from the above represents an infracted tissue, and the lower quarter represents the background region. This data is used to validate the proposed method. The imaging parameters used in simulation are: The slice selection is $10 \mathrm{~mm}$, matrix size is $100 \mathrm{X} 100$, low and high tuning frequencies are $0.2 \mathrm{~mm}^{-1}$ and $0.3 \mathrm{~mm}^{-1}$, respectively, and the initial tagging frequency is $0.21 \mathrm{~mm}^{-1}$. Human volunteer is scanned on Philips Acheiva 3T MR scanner with same imaging parameters of the simulation. C-SENC images are acquired 10-15 min post contrast agent injection [7]. The simulated and the Real C-SENC images are used to construct the input data, that is fed into the classification block to classify the different components in the image. Fig. 3, and Fig. 4 show simulated and real C-SENC images.

\subsection{Simulated Data Results}

We have chosen 6 popular classifiers, multilayer perceptron (MLP), support vector machine (SVM), radial basis function (RBF), decision trees (DT), bayes classifier, and simple k-means clustering technique. All implementation are carried out using the
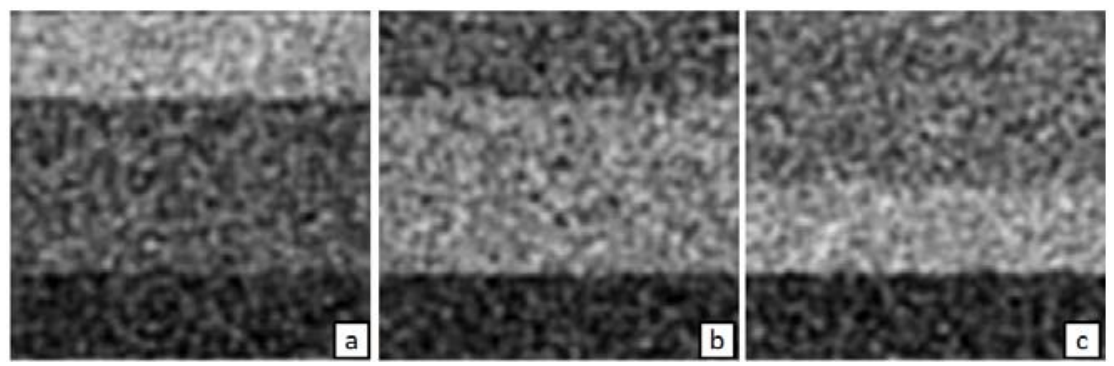

Fig. 3. Simulated (a) high-tune, (b) low-tune, (c) T1-weighted C-SENC image 

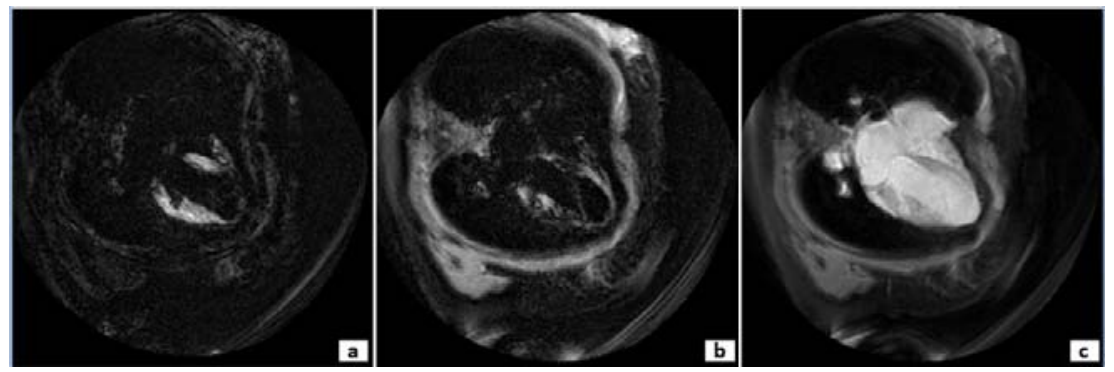

Fig. 4. Real (a) high-tune, (b) low-tune, (c) T1-weighted C-SENC image

WEKA library [8]. For all the supervised learning techniques, we use 10 fold cross validation and the default parameters that are set in WEKA. After constructing the input vector from the simulated images, we feed this data into the classifier block.

In case of MLP, the time taken to build the model is 43.27 seconds. Table 1 . shows the confusion matrix. The correctly identified regions are $87.09 \%$ while, $12.91 \%$ are wrongly classified.

Table 1. The confusion matrix when using multilayer perceptron with 10 folds cross validation

\begin{tabular}{|c|c|c|c|c|}
\hline & Healthy & Hibernating & Infarcted & Background \\
\hline Healthy & $\mathbf{2 3 5 4}$ & 96 & 35 & 14 \\
\hline Hibernating & 83 & $\mathbf{1 9 5 3}$ & 438 & 27 \\
\hline Infarcted & 39 & 516 & $\mathbf{1 9 4 3}$ & 1 \\
\hline Background & 4 & 35 & 3 & $\mathbf{2 4 5 9}$ \\
\hline
\end{tabular}

Support vector machine (SVM) is also used, where the time taken to build the model is 1.02 seconds. Table. 2 shows the confusion matrix. The correctly identified regions are $87.77 \%$ while, $12.23 \%$ are wrongly classified.

Table 2. The confusion matrix when using SVM with 10 folds cross validation

\begin{tabular}{|c|c|c|c|c|}
\hline & Healthy & Hibernating & Infarcted & Background \\
\hline Healthy & $\mathbf{2 3 7 9}$ & 77 & 32 & 11 \\
\hline Hibernating & 94 & $\mathbf{1 9 0 6}$ & 472 & 29 \\
\hline Infarcted & 46 & 427 & $\mathbf{2 0 2 5}$ & 1 \\
\hline Background & 5 & 26 & 3 & $\mathbf{2 4 6 7}$ \\
\hline
\end{tabular}

J48 decision tree is also used, where the time taken to build the model is 0.61 seconds. The constructed tree size is 131 with 66 leaf nodes. Table. 3 shows the confusion matrix. The correctly identified regions are $87.02 \%$ while, $13.98 \%$ are wrongly classified. 
Table 3. The confusion matrix when using J48 DT with 10 folds cross validation

\begin{tabular}{|c|c|c|c|c|}
\hline & Healthy & Hibernating & Infarcted & Background \\
\hline Healthy & $\mathbf{2 3 2 4}$ & 109 & 44 & 22 \\
\hline Hibernating & 114 & $\mathbf{1 9 8 8}$ & 384 & 15 \\
\hline Infarcted & 50 & 497 & $\mathbf{1 9 5 0}$ & 2 \\
\hline Background & 25 & 23 & 2 & $\mathbf{2 4 5 1}$ \\
\hline
\end{tabular}

Radial basis function (RBF) is also used, where the time taken to build the model is 9.41 seconds. Table. 4 shows the confusion matrix. The correctly identified regions are $87.49 \%$ while, $12.51 \%$ are wrongly classified.

Table 4. The confusion matrix when using RBF with 10 folds cross validation

\begin{tabular}{|c|c|c|c|c|}
\hline & Healthy & Hibernating & Infarcted & Background \\
\hline Healthy & $\mathbf{2 3 7 5}$ & 80 & 34 & 10 \\
\hline Hibernating & 92 & $\mathbf{1 9 0 4}$ & 475 & 30 \\
\hline Infarcted & 39 & 448 & $\mathbf{2 0 1 1}$ & 1 \\
\hline Background & 12 & 27 & 3 & $\mathbf{2 4 5 9}$ \\
\hline
\end{tabular}

Naive Bayes classifier is also used, where the time taken to build the model is 0.09 seconds. Table. 5 shows the confusion matrix. The correctly identified regions are $87.57 \%$ while, $12.43 \%$ are wrongly classified.

Table 5. The confusion matrix when using Bayes classifier with 10 folds cross validation

\begin{tabular}{|c|c|c|c|c|}
\hline & Healthy & Hibernating & Infarcted & Background \\
\hline Healthy & $\mathbf{2 3 8 9}$ & 73 & 28 & 9 \\
\hline Hibernating & 106 & $\mathbf{1 9 0 2}$ & 470 & 23 \\
\hline Infarcted & 53 & 428 & $\mathbf{2 0 1 8}$ & 0 \\
\hline Background & 9 & 41 & 23 & $\mathbf{2 4 4 8}$ \\
\hline
\end{tabular}

Finally, unsupervised machine learning technique, simple k-means (SKM), is used where the Euclidean distance is used as a distance function. Table. 6 shows the confusion matrix. The correctly clustered regions are $84.44 \%$ while, $15.56 \%$ are wrongly clustered.

Table 6. The confusion matrix when using SKM

\begin{tabular}{|c|c|c|c|c|}
\hline & Healthy & Hibernating & Infarcted & Background \\
\hline Healthy & $\mathbf{2 3 7 1}$ & 84 & 27 & 17 \\
\hline Hibernating & 108 & $\mathbf{1 8 7 6}$ & 480 & 37 \\
\hline Infarcted & 60 & 412 & $\mathbf{2 0 2 6}$ & 1 \\
\hline Background & 4 & 20 & 2 & $\mathbf{2 4 7 5}$ \\
\hline
\end{tabular}

In case of using the SKM algorithm, no time is needed to build a model. It is the fastest technique in the proposed techniques. But, as stated in section 3, labeling stage is needed when using unsupervised learning technique. 


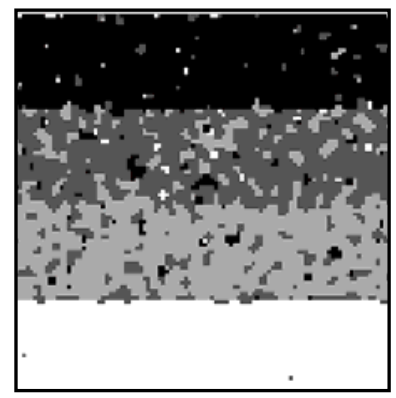

Fig. 5. The result after applying SKM on the simulated C-SENC images

Fig. 5 shows the result after applying SKM on the simulated C-SENC images. As shown in the figure it was able to cluster the 4 regions in the simulated images. Also, we can notice that there are misclustered pixels.

From the previous results, we notice that the majority in the wrongly classified or clustered regions is in the infracted and hibernating regions, and this is due to the near characteristics between those regions, since they are nearly have the same pattern in the low-and high tune images, and slightly differs in the no-tune image as shown in the simulated image.

After testing all the previous techniques, we found that SVM, Naive Bayes classifier, and SKM are the best classifiers in terms of time and accuracy. Although the MLP has a good accuracy but it is time consuming, also J48 DT has a good accuracy but the tree size is large.

\subsection{Real MR Data Results}

Real C-SENC images are acquired, and samples from different regions are manually selected to test the SVM, Naive Bayes and SKM classification techniques. Fig. 6 shows the different regions that are selected from the image.

For simplicity, 3 regions only are selected, healthy, infracted and background, as in real MR images, if there is a hibernating tissue, it just appear in a very fine region. And it is practically impossible to determine hibernating tissues visually.

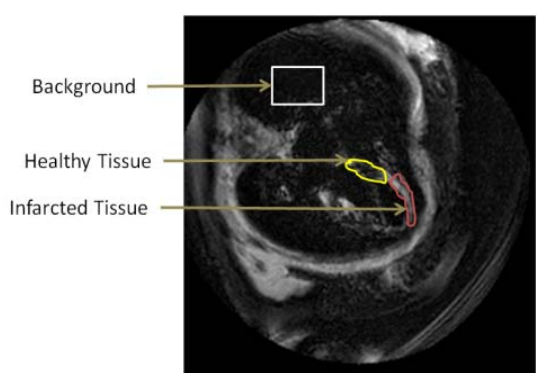

Fig. 6. Different selected regions represent healthy, infracted tissue and background 
Table 7. The confusion matrix when using Bayes classifier with 10 folds cross validation

\begin{tabular}{|c|c|c|c|}
\hline & Background & Healthy & Infarcted \\
\hline Background & $\mathbf{5 5 5}$ & 0 & 0 \\
\hline Healthy & 0 & $\mathbf{1 6 8}$ & 47 \\
\hline Infarcted & 0 & 8 & $\mathbf{2 5 6}$ \\
\hline
\end{tabular}

Firstly, Naive Bayes algorithm with 10 fold cross validation is used. Table. 7 shows the confusion matrix. The correctly identified regions are $95.04 \%$ while, $4.95 \%$ are wrongly classified.

SVM with 10 fold cross validation is also used, where the time taken to build the model is 0.66 seconds. Table. 8 shows the confusion matrix. The correctly identified regions are $93.59 \%$ while, $6.4 \%$ are wrongly classified.

Table 8. The confusion matrix when using SVM with 10 folds cross validation

\begin{tabular}{|c|c|c|c|}
\hline & Background & Healthy & Infarcted \\
\hline Background & $\mathbf{5 5 5}$ & 0 & 0 \\
\hline Healthy & 0 & $\mathbf{1 6 3}$ & 48 \\
\hline Infarcted & 10 & 8 & $\mathbf{2 4 6}$ \\
\hline
\end{tabular}

Finally, simple k-means (SKM) is used and table. 9 shows the confusion matrix. The correctly clustered regions are $88.55 \%$ while, $11.45 \%$ are wrongly clustered.

Table 9. The confusion matrix when using SKM

\begin{tabular}{|c|c|c|c|}
\hline & Background & Healthy & Infarcted \\
\hline Background & $\mathbf{5 5 5}$ & 0 & 0 \\
\hline Healthy & 0 & $\mathbf{1 4 1}$ & 70 \\
\hline Infarcted & 44 & 4 & $\mathbf{2 1 6}$ \\
\hline
\end{tabular}
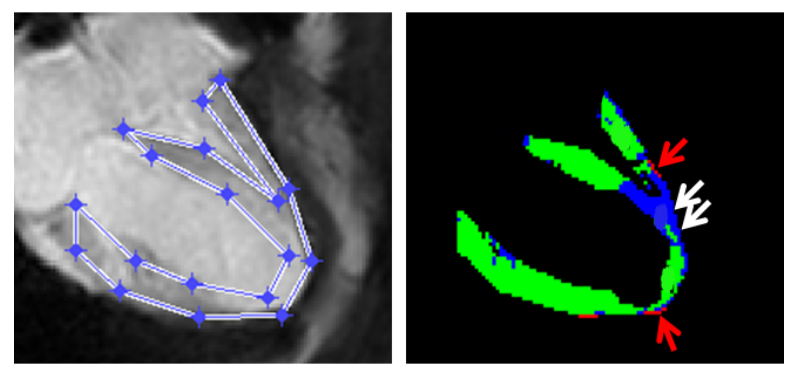

Fig. 7. The myocardium has been clustered to three different types of tissues 
Fig. 7 shows a long-axis view of the heart where we define a region of interest in the heart muscle (myocardium). The myocardium is clustered to different groups, healthy, infracted and hibernating tissues. The white arrows point to potentially infracted regions, while the red arrows point to potentially hibernating regions. The healthy regions of the myocardium appear in green color.

\section{Summary and Conclusion}

In conclusion, in this work, we attempt to investigate machine learning techniques like MLP, SVM, Bayesian, RBF, DTs and SKM to identify the different heart tissues and background in C-SENC cardiac images. The proposed techniques allows for objectively identifying divergent heart tissues, which would be potentially important for clinical decision-making in patients with MI. The proposed method is tested using numerical simulations of the heart C-SENC images of MI and real images of patients. The results show that the proposed techniques are able to identify the different components of the image with a high accuracy. The proposed techniques vary in the computation time and accuracy. In our opinion using SVM, Bayes classifiers gives the best results, as they have high accuracy and low computation time. The misclassified instances is due to the poor SNR, $21 \mathrm{~dB}$, however; recently new imaging techniques are proposed, where better quality images are obtained, which enhance the classification results.

\section{Acknowledgment}

This work is supported by PDP grant from ITIDA agency, Ministry of Communication and Information technology, Egypt. We would like to thank Eng. Esraa and Eng. Rana for their valuable discussion.

\section{References}

1. Ibrahim, E.-S.H., Stuber, M., Kraitchman, D.L., Weiss, R.G., Osman, N.F.: Combined Functional and Viability Cardiac MR Imaging in a Single Breathhold. Magn. Reson. Med. 58, 843-849 (2007)

2. Watzinger, N., Saeed, M., Wendland, M.F., Akbari, H., Lund, G., Higgins, C.B.: Myocardial viability: magnetic resonance assessment of functional reserve and tissue characterization. J. Cardiovasc. Magn. Reson. 3, 195-208 (2001)

3. Kim, R.J., Wu, E., Rafael, A., Chen, E., Parker, M.A., Simonetti, O., Klocke, F.J., Bonow, R.O., Judd, R.M.: The use of contrast-enhanced magnetic resonance imaging to identify reversible myocardial dysfunction. N. Engl. J. Med. 343, 1445-1453 (2000)

4. Osman, N.F., Sampath, S., Atalar, E., Prince, J.L.: Imaging longitudinal cardiac strain on short-axis images using strain-encoding MRI. Magn. Reson. Med. 46, 324-334 (2001)

5. Ibrahim, E.-S.H., Weiss, R.G., Stuber, M., Spooner, A.E., Osman, N.F.: Identification of Different Heart Tissues from MRI C-SENC Images Using an unsupervised Multi-Stage Fuzzy Clustering Technique. J. Magn. Reson. Imaging 28(2), 519-526 (2008) 
6. Motaal, A.G., El Gayar, N., Osman, N.F.: Automated Cardiac-Tissue Identification in Composite Strain-Encoded (C-SENC) Images Using Fuzzy C-means and Bayesian Classifier. Accepted in 4th International Conference on Bioinformatics and Biomedical Engineering (iCBBE 2010), Chengdu, China (2010)

7. Sharma, P., Socolow, J., Patel, S., Pettigrew, R., Oshinski, J.: Effect of Gd-DTPA-BMA on blood and myocardial $\mathrm{T} 1$ at $1.5 \mathrm{~T}$ and $3 \mathrm{~T}$ in humans. Magn. Reson. Imaging 23, 323-330 (2006)

8. Witten, L.I., Frank, E.: Data Mining: Practical Machine Learning Tools and Techniques with Java Implmentations. Morgan Kaufmann, San Francisco (1999) 